immunohistochemistry (POLEmt: POLE mutated; MMRd: mismatch repair deficient, p53abn: p53+) represent the main pathologic features to define the prognostic risk groups in endometrial cancer. This study aimed to evaluate the correlation between the prognostic factors and the rate of recurrence in our experience.

Methodology We conducted a monocentric retrospective study of consecutive women who underwent surgical treatment for endometrial cancer at Department of Gynecology Oncology, Treviso Regional Hospital, between January-2017 to October2020. Baseline demographics, histopathologic type, grading, FIGO stage, LVSI, myometrial infiltration, lymph node involvement (LNI), and immunohistochemistry (when available) were collected. The diagnosis of recurrence and his pattern (distant or pelvic) were analyzed on the basis of prognostic factors type.

Result(s)* A total of 146 women were included: 105 patients in stage I (72\%), 15 in stage II (10\%), 22 in stage III (15\%) and 4 in stage IV (3\%). The rate of recurrence was 13\% (19 patients) with a median time to recurrence of 15 months (342). A univariate approach showed a significantly association between recurrence and histopathologic non-endometrioid type (O.R 3.13; p=0.05), LNI (O.R 4.05; p=0.03), myometrial infiltration (O.R. 2.84; $\mathrm{p}=0.04)$, positive-LVSI (O.R. $3.32 ; \mathrm{p}=0.01$ ), grading (O.R. 3.9; $\mathrm{p}=0,0001)$, FIGO-stage (II O.R. 1.64; III O.R. 2.91; IV O.R. 10.20; $\mathrm{p}=0.03)$, and p53abn (O.R. 5.10; p=0.02). We found no significant differences between recurrence and MMRd (O.R. 0.90; p:0,8), no POLEmt was found in our recurrence patients. A positiveLVSI related with a distant recurrence (O.R 8.00; $\mathrm{p}=0.003$ ). In multivariate analysis, FIGO stage and p53abn $(\mathrm{OR}=1.1$ and $\mathrm{OR}=5.3$; $\mathrm{p}$-value $=0.05$, respectively) were found independent predictors factor for recurrence. In particular, all patients with p53abn and recurrence were in FIGO stage I at the diagnosis.

Conclusion* All prognostic factors related to recurrence, and FIGO-stage represented the most significant risk factor. Instead, when we considered immunohistochemistry, p53abn resulted in a negative prognostic factor for recurrence, independent to FIGO-stage. Positive-LVSI presented an important correlation with distant recurrence compared to women with negative-LVSI and only pelvic recurrences. Risk factors are important to define prognostic risk groups and adjuvant therapy.

\section{SURVIVAL OF ELDERLY PATIENTS WITH ENDOMETRIAL CANCER - PREDICTED BY PREOPERATIVE G-8 GERIATRIC SCREENING TOOL}

${ }^{1} \mathrm{~K}$ Anic*, ${ }^{1} \mathrm{C}$ Altehoefer, ${ }^{1} \mathrm{MW}$ Schmidt, ${ }^{1} \mathrm{~S}$ Krajnak, ${ }^{1} \mathrm{R}$ Schwab, ${ }^{1} \mathrm{~V}$ Linz, ${ }^{2} \mathrm{C}$ Westphalen, ${ }^{3}$ EK Hartmann, ${ }^{1} \mathrm{M}$ Schmidt, ${ }^{1} \mathrm{~A}$ Hasenburg, ${ }^{1} \mathrm{M}$ Battista. ${ }^{1}$ University Medical Centre of the Johannes Gutenberg University Mainz, Department of Gynaecology and Obstetrics, Mainz, GERMANY; '2University Medical Centre of the Johannes Gutenberg University Mainz, Department of Geriatric Medicine, Mainz, GERMANY; ${ }^{3}$ University Medical Centre of the Johannes Gutenberg University Mainz, Department of Anesthesiology, Mainz, GERMANY

\subsection{6/ijgc-2021-ESG0.128}

Introduction/Background* We evaluated the prognostic impact of various global health assessment tools in accordance to conventional prognostic factors in patients with endometrial cancer (EC) older than 60 years.
Methodology G-8 geriatric screening tool (G-8 geriatric score), Lee Schonberg prognostic index (Lee-Index), Charlson Comorbidity Index (CCI) and American Society of Anesthesiologists (ASA-PS) - Physical Status System were retrospectively determined in a consecutive cohort of elderly patients with EC. Univariate and multivariate Cox regression analyses and Kaplan-Meier method were performed to determine the impact of the global health assessment tools on progression free survival (PFS) and overall survival (OS).

Result(s)* 153 patients entered the study. In multivariate analysis adjusted for common clinical-pathological risk factors (e.g. histological type and stadium, histological grading, FIGO-stadium, tumor stadium and postoperative tumor burden) and different global health assessment tools (G-8 geriatric score, Lee-Index, CCI and ASA-PS) only the G-8 geriatric score retained its significance as a considerable and independent prognostic factor of 5-year OS rate (HR: 3.173; 95\%-CI [1.436-7.010]; $\mathrm{p}=0.004)$ but not for univariate 5-year PFS rate (HR: 2.033; 95\%-CI [0.925-4.468]; p=0.078). 92 patients $(61.3 \%)$ were assigned to the G-8-non-frail cohort (cut-off value $>14$ points) with an increased 5 -year PFS and OS rate compared to the 58 patients $(38.7 \%)$ classified as G8 -frail (PFS: $82.1 \%$ vs. $65.4 \%$; $\mathrm{p}=0.071$ and OS: $88.2 \%$ vs. 49.7\%; $<<0.000$; respectively).

Conclusion* Preoperative G-8 geriatric score independently predicted 5-year OS in elderly EC patients irrespectively of the maximal surgical effort.

\section{TYPE OF SENTINEL LYMPH NODES METASTASES AND ONCOLOGIC OUTCOMES IN ENDOMETRIAL CANCER PATIENTS: AN ITALIAN MULTI-INSTITUTIONAL STUDY}

${ }^{1} \mathrm{~F}$ Martinelli* ${ }^{*}{ }^{2} \mathrm{~A}$ Buda, ${ }^{3} \mathrm{~F}$ Fanfani, ${ }^{4} \mathrm{~F}$ Legge, ${ }^{5} \mathrm{M}$ Roccio, ${ }^{6} \mathrm{~F}$ Falcone, ${ }^{7} \mathrm{AM}$ Perrone, ${ }^{8}$ J Casarin, ${ }^{9} \mathrm{~A}$ Perutelli, ${ }^{10} \mathrm{~N}$ Biglia, ${ }^{11} \mathrm{P}$ Scollo, ${ }^{12} \mathrm{~F}$ Romano, ${ }^{1} \mathrm{~A}$ Ditto, ${ }^{2} \mathrm{D}$ Ferrari, ${ }^{3} \mathrm{G}$ Monterossi, ${ }^{4} \mathrm{~F}$ Murgia, ${ }^{2} \mathrm{C}$ Paniga, ${ }^{3} \mathrm{G}$ Scambia, ${ }^{1} \mathrm{~F}$ Raspagliesi. ${ }^{1}$ Fondazione IRCCS Istituto Nazionale Tumori of Milan, Gynecologic Oncology, Milan, Italy; ${ }^{2}$ University of Milan Bicocca, UOC of Gynecology A.O. San Gerardo, Clinic of Obstetrics and Gynecology; ${ }^{3}$ Fondazione Policlinico Universitario A. Gemelli Università Cattolica del Sacro Cuore; ${ }^{4}$ Ospedale Generale Regionale 'F.Miulli'; ${ }^{5}$ Fondazione IRCCS Policlinico San Matteo, U.O. di Ostetricia e Ginecologia; ${ }^{6}$ Endoscopica Malzoni, Center for Advanced Endoscopic Gynecological Surgery; ${ }^{7}$ RCCS Azienda Ospedaliero-Universitaria di Bologna, Division of Oncologic Gynecology; ${ }^{8}$ University of Insubria, Obstetrics and Gynecology Department Del Ponte Women's and Children Hospital; ${ }^{9} \mathrm{UO}$ ginecologia 2 di Pisa; ${ }^{10}$ Università di Torino SCDU Ginecologia e Ostetricia A. O. Ordine Mauriziano Umberto I; ${ }^{11}$ Ospedale Cannizzaro, AO per I'Emergenza; ${ }^{12}$ Institute for Maternal and Child Health 'IRCCS Burlo Garofolo', Trieste

\subsection{6/ijgc-2021-ESGO.129}

Introduction/Background* The role of volume of sentinel lymph nodes (SLNs) disease (macro-micrometastases and ITCs) in endometrial cancer is not clearly defined. We aimed to asses predictive factors for SLNs involvement and recurrence free survival (RFS) in patients with endometrial cancer.

Methodology A multicenter retrospective evaluation of endometrial cancer patients with positive (macro-micro metastases or ITCs) SLNs, treated between 2003 and 2020, was performed. Predictive factors for nodal involvement (endometrioid vs non-endometrioid histology, grading, lymphovascularspace invasion (LVSI), myometrial invasion (MI), cervical stromal invasion, ESGO/ESTRO/ESP risk group), adjuvant therapy and oncological outcomes were evaluated. 